Reprinted from the Journal of Chemical Physics, Vol. 36, No. 9, 2518-2519, May 1, 1962 Printed in U. S. A.

\title{
Spin Exchange between Excitons and Free Radicals*
}

\author{
H. M. MCConneli, $\dagger$ H. O. Griffith, $\ddagger$ AND D. PoOLEy \\ Gates and Crellin Laboratories of Chemistry, II \\ California Institute of Technology, Pasadena 4, California
}

(Received January 8, 1962)

$\mathbf{I}^{\mathrm{N}}$ $\mathrm{N}$ previous work it has been suggested that the lowtemperature thermally excited electronic states of many aromatic "free radical" solids may be triplet $(S=1)$ exciton states. ${ }^{1}$ The expected ${ }^{2}$ spin resonance

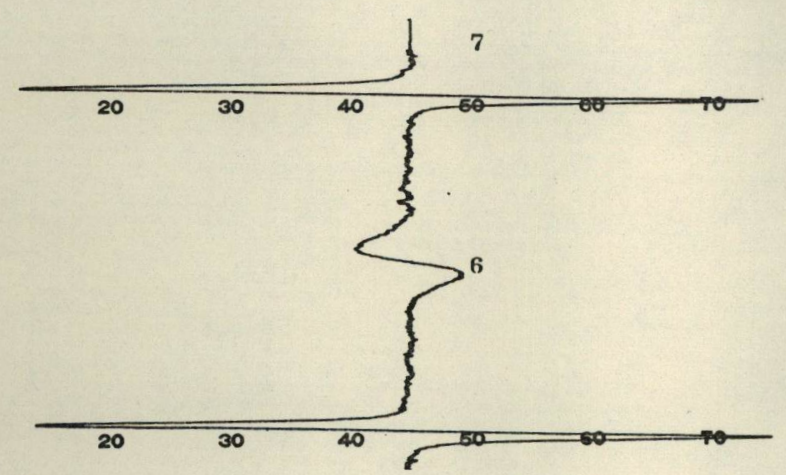

(a)

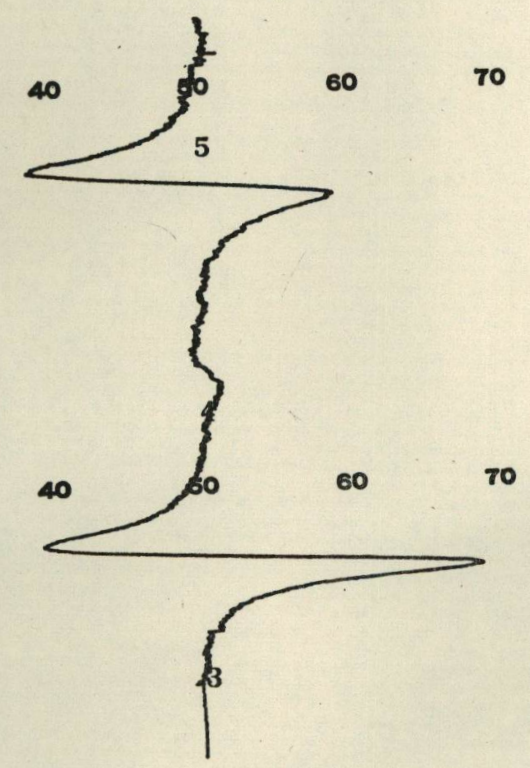

(b)

Fig. 1. Exciton and free radical resonances in

$$
\left(\phi_{3} \mathrm{PCH}_{3}\right)+(\mathrm{TCNQ})_{2}^{-} \text {. }
$$

(a) Exciton (outer) and free radical (center) resonance at $\sim-150^{\circ} \mathrm{C}$, where the exciton-free radical spin exchange rate is small. The fine structure splitting is $175 \mathrm{Mc}$. (b) Spin exchange broadening of the exciton and free radical resonances in the $\sim 105-125^{\circ} \mathrm{C}$ range where the exciton concentration is of the order of a percent. The apparent fine structure splitting is 165 Mc [same crystal orientation as (a)], and the apparent asymmetry of left- and right-hand peaks arises from a $\sim 20^{\circ} \mathrm{C}$ crystal warmup during the period required to scan the spectrum. characteristics of triplet exciton states-vanishing nuclear hyperfine structure, and nonvanishing fine structure at low exciton concentrations, and exchange interactions between excitons - appear to have been observed in some charge-transfer TCNQ free-radical solids by Chestnut and Phillips, ${ }^{3}$ and may also have been observed by investigators at Stanford in a number of other free-radical solids. ${ }^{4}$ In the present note we report a new experiment that supports the idea that the triplet excitations are mobile.

A single crystal of $\left(\phi_{3} \mathrm{PCH}_{3}\right)+(\mathrm{TCNQ})_{2}{ }^{-}$kindly given to us by Chestnut and Phillips was $\mathrm{x}$ irradiated and subsequently showed the low-temperature $\left(\sim-150^{\circ} \mathrm{C}\right)$ spin resonance spectrum illustrated in Fig. 1(a). The two narrow lines have been interpreted previously as a fine structure splitting of an exciton state, ${ }^{1}$ and the broader line is the $\mathrm{x}$-ray produced free radical..$^{5}$ Figure 1 (b) shows the spectrum of the same sample at a higher temperature $\left(\sim-115^{\circ} \mathrm{C}\right)$ where the relative tripletstate concentration (triplet/singlet) is higher of the order of a percent. The broadening of the fine-structure doublet (in undamaged samples) has already been attributed to exchange interactions between triplet states, ${ }^{3}$ and in particular has been attributed to spin exchange collisions between mobile triplet excitons. ${ }^{1}$ Since exchange interaction between triplet states is not the only conceivable mechanism for the loss of fine structure splitting, it is significant that in Fig. 1(b) the $S=\frac{1}{2}$ free radical signal undergoes a comparable broadening, which in this case must almost certainly be due to exciton-free radical spin exchange. It is therefore likely that in undamaged samples the broadening and loss of the fine structure splitting is also due to spin exchange interactions between excitons.

The present experimental results further support the idea that the triplet excitons are mobile. That is, the free radical signal undergoes a marked and apparently homogeneous broadening at an exciton concentration of the order of a percent. Immobile and localized triplet excitations could hardly account for this broadening of the entire $S=\frac{1}{2}$ free radical signal. On the other hand, the observed free radical exchange broadening is quite compatible with the idea of highly mobile excitations, in which case the exciton-free radical exchange interaction is approximately proportional to the exciton concentration and a nearest neighbor (free-radical)-(triplet state) exchange integral.

Further studies of radiation damage in these and 
other paramagnetic crystals are in progress. We are greatly indebted to D. B. Chestnut, W. D. Phillips, and R. E. Merrifield for helpful discussions.

* Sponsored by the National Science Foundation.

$\dagger$ Alfred P. Sloan Fellow.

$\$$ National Science Foundation Predoctoral Fellow.

$\S$ A. A. Noyes Fellow.

II Contribution No. 2802.

${ }^{1}$ H.M. McConnell and R. Lynden-Bell, J. Chem. Phys. 36, 2393

${ }^{2}$ H. Ster

(1961).
${ }^{3}$ D. B. Chesnut and W. D. Phillips, J. Chem. Phys. 35, 1002 (1961).

${ }^{4}$ R. S. Rhodes, J. H. Burgess, and A. S. Edelstein, Phys. Rev. Letters 6, 462 (1961); M. E. Anderson, R. S. Rhodes, and G. E. Pake, J. Chem. Phys. 35, 1527 (1961); R. H. Webb, Phys. Rev. Letters 6, 611 (1961); A. S. Edelstein and M. Mandel, J. Chem.
Phys. 35, 1130 (1961).

${ }^{5}$ The identity of the free radical(s?) is not known at present. The close similarity of the free radical $g$ factor at selected crystal orientations to that of the exciton $(\Delta g \lesssim \pm 0.0005)$ suggests (TCNQ) $)^{-}$, or (TCNQ) ${ }_{n}^{-}$, where $n$ is a small integer. However at certain crystal orientations, weak free radical fine structure splittings are observed that are somewhat similar to those we find in $\mathrm{x}$-irradiated $\left(\phi_{3} \mathrm{PCH}_{3}\right)+\mathrm{I}^{-}$. 